\title{
The Humean pragmatic turn and the case for revisionary best systems accounts
}

\author{
Toby Friend ${ }^{1} \mathbb{D}$
}

Received: 25 May 2021 / Accepted: 3 December 2021 / Published online: 28 January 2022

(c) The Author(s) 2022

\begin{abstract}
Lewis's original Best Systems Account of laws was not motivated much by pragmatics. But recent commentary on his general approach to laws has taken a "pragmatic turn'. This was initiated by Hall's defence against the charge of 'ratbag idealism' which maintained that best systems accounts should be admired rather than criticised for the inherent pragmatism behind their choice of desiderata for what counts as 'best'. Emboldened by Hall's pragmatic turn, recent commentators have proposed the addition of pragmatically motivated desiderata to complement or replace the canonical desiderata of strength and simplicity. This, they hope, will allow their revisionary BSAs to respond better to various counterexamples against the original account. While the pragmatic turn itself is well taken, here I problematise these revisionary approaches. First, there are reasonable responses to the counterexamples from within the canonical BSA. Second, while actual laws may satisfy the newly proposed desiderata, there are reasons to think these desiderata cannot be constitutive of laws. By comparison, the canonical desiderata appear to be relevant to explaining why and when the revisionary desiderata will reflect pragmatic features of the laws and better reflect the motives behind practitioners of fundamental physics.
\end{abstract}

Keywords Best system account of laws $\cdot$ Humeanism $\cdot$ Pragmatics $\cdot$ Revision

\section{Introduction}

Knowledge of the laws of nature confers upon us a great deal of practical ability. It enables us to predict, manipulate, construct, avoid and generally comprehend much about the world. It would be more than curious if there was no explanation of that fact; something about laws explains why knowledge of them is able to facilitate this.

Recent commentary on the Humean Best Systems Account (BSA) of laws has brought closer attention to the question of laws' practical value. The Humean is

Toby Friend

toby.friend@bristol.ac.uk

1 Department of Philosophy, University Bristol, Cotham House, BS6 6JL Bristol, UK 
well-placed to make sense of the close connection between practical value and lawhood, since they are able, if they so choose, to give a reductive account in terms of pragmatically motivated criteria. However, it has been repeatedly observed that the canonical or 'official guiding idea' behind the BSA cannot alone account for many of the practical features laws actually have or could have had. This has led some to suggest a departure from the canonical BSA by substituting or embellishing its desiderata of simplicity and strength with others tailored to making sense of these features (Hall, 2015; Hicks, 2018; Dorst, 2019; Jaag \& Loew, 2020).

It is my aim to show that much of the reasoning behind these revisions to the canonical BSA is misguided. I do not claim that the newly identified desiderata aren't genuine features of laws nor that they signify laws' practical utility. Nevertheless, my claim is (first) that there are reasonable responses to the criticisms and counterexamples which have been used to motivate revision on behalf of the canonical account. Moreover, I also claim (second) that unlike the canonical desiderata, it is implausible to think of the newly proffered desiderata as being constitutive of laws. Laws shouldn't necessarily be expected to exhibit these practical features, and whether they do or not is very plausibly explained by whether they are theorems of the canonical best system. ${ }^{1}$

Here's the plan. Section 2 introduces the best systems approach to laws with an emphasis on getting the order of explanatory priorities right. Section 3 discusses a complaint, and Hall's response, that the approach renders laws constitutively pragmatic. Hall's response is that the pragmatics should actually be seen as a benefit of the approach. This marks the 'pragmatic turn' in BSAs. Section 4 then surveys three revisionary approaches to BSA motivated by putative counterexamples to the canonical approach which employ further pragmatic desiderata to address them. Section 5 offers some reasonable responses to these counterexamples on behalf of the canonical BSA. Section 6 then investigates the plausibility of the proffered revisionary desiderata by exploring whether or not they assist either in getting us what we want from laws (Section 6.1), by making laws easier to access (Section 6.2), or by making laws easier to use (Section 6.3). I argue that they do not obviously help in any of these ways. Section 7 concludes.

Before getting things underway, a necessary qualification is in order. In all that follows, I'll be concerned specifically with the project of employing some or other desiderata to chose those systems which give us the laws of physics. Although it is rarely made explicitly among revisionists, the issues which motivate the addition of further desiderata and the proffered desiderata themselves are almost always comprehended within the context of physical theory. Hall's initial discussion was clearly oriented towards physics, highlighted by the example of the 'limited oracular perfect physicist' and emphasis on the distinction between weak constraints on initial conditions and strong dynamical constraints; Hicks's (2018) alternative force law, and examples of modularity and breadth in the laws are all in the remit of (possible) physics; Dorst's (2019) desiderata (of symmetry, locality and informative dynamics)

\footnotetext{
${ }^{1}$ Demarest (How (Not) to be a Pragmatic Humean, unpublished MS) has recently and usefully put this in terms of a distinction between what counts as an analysis of laws and what counts merely as capturing the role laws play for us.
} 
also clearly indicates that the relevant science under discussion is physics. Humeans (even revisionists) are often and rightly interested in how the special science laws are to be retrieved from systematisation beyond those of physics. But I take this to be a discussion which goes beyond the debate over the need for revisionary desiderata. Or at least, none of the proffered desiderata to be discussed below come from a desire to retrieve the special science laws more effectively (contrast this with the discussions in Loewer, 2012; Cohen \& Callender, 2009; Schrenk, 2014).

\section{BSA and Euthryphro-style questions}

In discussing laws of nature, Lange (2009) asks an analogue to a question posed by Socrates to Euthyphro: are the laws necessary because they are laws, or are they laws (in part) because they are necessary? The 'Euthyphro-style' question assumes that laws exhibit some degree of necessity and enquires after the explanatory order of priority between laws and necessity. And there are other widely agreed upon features of laws to which further Euthyphro-style questions can be asked. For any characteristic $X$ of laws, be it their comprehensiveness, simplicity or practical utility, we can ask 'do the laws exhibit $X$ because they are laws, or are they laws (in part) because they exhibit $X$ ?'

Humeans have traditionally been fairly unanimous in their answers to many of these questions. For them, the world lacks worldly necessary connections, so laws' necessity is something to be 'added in' (by us) after their legality has been established. Specifically, there is widespread agreement that it is constitutive of being a law at any world that it be a regularity which satisfies, or best satisfies, some or other non-modally specified criterion distinguishing it from non-lawlike regularities. Humeans have argued, for instance, that what marks out generalisations of fact from generalisations of law 'lies not so much on the side of facts which make them true or false, as in the attitude of those who put them forward' (Ayer, 1956), where, for instance, something 'is called a law because it is used for prediction' as opposed to 'being used for prediction because it is a law' (Goodman, 1983, 26).

An evident worry is that demarcating the laws by how they're used risks putting their status down to the whims of whoever is using them. At least since Lewis (1973) (although the idea was prescient in Mill and Ramsey) many Humeans have obviated this concern by employment of a 'best systems account' (henceforth BSA) of laws: according to the canonical version, the laws of a world are the generalisations (a statement of a regularity) of any true deductively closed system which is objectively maximally pithy, achieving a best combination of comprehensiveness, or 'strength', and conciseness, or 'simplicity' (Lewis, 1973, 1983, 1994; BraddonMitchell, 2001; Loewer, 1996, 2007a; Cohen \& Callender, 2009; Schrenk, 2014). ${ }^{2}$

\footnotetext{
${ }^{2}$ I follow the trend of leaving aside discussion of the sometimes-invoked criterion of 'fit' used to accommodate probabilistic laws. Whether or not it is included in the canonical BSA is irrelevant to the discussion here.
} 
For Humeans who adopt this 'canonical BSA' it is a (statement of a) regularity's inclusion in a deductive system which achieves the best compromise of these desiderata which explains why it is used for prediction, and so why it deserves to be called a law. The canonical BSA therefore settles an explanatory ordering for characteristics of laws generated in response to Euthyphro-style questions, which we might display as follows:

$$
\text { Strength \& Simplicity } \Rightarrow \text { Laws } \Rightarrow \text { Necessity }
$$

(Laws are necessary because they are laws, but they are laws because they follow from a system which achieves a best compromise of simplicity and strength.)

\section{The pragmatic turn}

The canonical desiderata of simplicity and strength certainly go some way to respond to the charge of anthropocentricity. But worries still remain. One issue concerns the language in which the systems are expressed. If we allow systems expressed in any language to compete then there is a trivial winner: the system $S$ comprising only the statement everything $F s$, where $F$ applies to all and only things at worlds where $S$ is true (Lewis, 1983, 367). To prohibit such triviality some restriction on the competing systems' languages must be made. Lewis's favoured view was to require that all systems be expressed in that language whose predicates denote only perfectly natural properties (where 'naturalness' is taken as a primitive). Others have suggested less metaphysically-loaded options (Earman, 1986; Loewer, 2007b; Cohen \& Callender, 2009; Eddon \& Meacham, 2015), and we may suppose some way or other the Humean has something plausible to say about this. ${ }^{3}$

The charge of anthropocentricity does not stop here, however. Lewis envisioned a 'ratbag idealist' who considers the laws hostage to the way we think and worried that his account might be criticised for such idealism, since according to it the laws are decided on the grounds of desiderata which are in some sense 'up to us'. Lewis's response to this problem was to assert the objectivity of strength and simplicity. ${ }^{4}$

It's not because of how we happen to think that a linear function is simpler than a quartic or a step function; it's not because of how we happen to think

\footnotetext{
${ }^{3}$ No doubt there is the potential for pragmatics to creep in at this stage, for instance in choosing a specific class of predicates (e.g. those which enable theories for space-time magnitude or motion and location; see Cohen \& Callender, 2009 for a discussion) with which systems may be formulated. However, a Humean can grant that without going so far as to require an expansion of the desiderata for choosing the law-giving system as the revisionist advises.

4 Nevertheless, Humeans may well have lingering concerns. Ultimately, some form of 'rigidification' to whatever sense of simplicity and informativeness is actually applied by us may be necessary (Eddon \& Meacham, 2015).
} 
that a shorter alternation of prenex quantifiers is simpler than a longer one; and so on. Maybe some of the exchange rates between aspects of simplicity, etc., are a psychological matter, but not just anything goes. (Lewis, 1994, 232)

I'll later (Section 5) suggest that Lewis may not have here captured the required notion of simplicity right. Arguably, however, the deeper issue is not so much whether or not there are objective standards of simplicity and strength, but with the fact that they are desiderata chosen purely for pragmatic reasons. Why should we think laws have anything to do with the practices we want to be engaged in? More recently, however, Hall (2015) has argued that the issue actually reveals a previously unemphasised benefit of the Humean account. It is unquestionable, Hall notes, that certain virtues rooted in what is of benefit to us knowing are at play in our theorybuilding, and so also in our positing of laws. Moreover, simplicity and comprehensiveness appear to be good candidates for such virtues. So all accounts of lawhood (Humean or otherwise) face the question of why those standards are conducive to discovering the laws, as indeed we think they are. Here the defender of BSA (canonical or otherwise) has a plausible answer, that it is constitutive of laws that they feature in those systems which have the virtues we aspire to in our theory-building (cf. Lewis, 1980, 123). Contrast this with some non-reductivist account according to which lawhood is explained in other terms. Under such accounts, the connection between epistemic virtues and laws remains unexplained.

As Hall acknowledges, his response to the charge of idealism places practical utility at the heart of any Humean account of laws.

[D]o not ask what, given such a metaphysical outlook, laws are. Ask instead how, given such a metaphysical outlook, one might usefully draw a distinction between certain facts about the world that are in some sense distinctively appropriate targets for scientific inquiry (DATSIs), and other facts that are less interesting and central [...] For now, I simply wish to note that there is plenty of room for the view that it is in part facts about us-idiosyncratically about us, and our peculiar human psychologies - that play a role in determining the most appropriate way for us to structure our investigation of the world. How could the details of our peculiar human situation not be relevant to this matter? And if so, then of course it should be partly "up to us"- that is, partly contingent on our particular needs and limitations as inquirers-what counts as a DATSI. $(2015,268)$

If Hall is right, it is in fact a boon for the canonical BSA if the desiderata of simplicity and strength are virtues defined by our anthropocentric needs.

Hall's response to the charge of idealism marks a 'pragmatic turn' in the Humean's approach to laws: laws are not pragmatic because they're laws, rather they're laws (in part) because they're pragmatic. Through the lens of the pragmatic turn the canonical BSA's order of explanatory priorities looks as follows.

$$
\text { Pragmatic utility } \Rightarrow \text { Strength } \& \text { Simplicity } \Rightarrow \text { Laws } \Rightarrow \text { Necessity }
$$




\section{The case for a revisionary BSA}

Hall's response to the charge of ratbag idealism has been widely endorsed (Hicks, 2018; Dorst, 2019; Jaag \& Loew, 2020, all acknowledge Hall's influence on their revisionary proposals). In effect, it reverses the complaint of anthropocentricity against BSA, bringing into question the idea that laws could be anything other than regularities favourable to our effective engagement with the world. However, given what science actually treats as distinctive targets for enquiry, the canonical BSA can still appear to be recommending the wrong regularities.

Hall (2015, 269-70) himself considers a 'phony constant': a single real number which encodes all the coordinates, masses, and charges of all particles at an instant by interleaving them systematically in decimal notation. If such a number were introduced to the best-achieving systems, Hall suggests, it would greatly increase its informativeness (at least in a deterministic world) without much compromise on simplicity. But we do not treat phony constants as laws. ${ }^{5}$ A related concern Hall raises is that there might be, or at least could have been, states whose physical features are by contingent fact exactly and simply summarised. Alongside deterministic generalisations such statements might contribute a lot in strength without much cost to simplicity, but again they are not, nor could they be, laws.

The straightforward response to these worries is that while the phony constant and simple summaries of physical state may go in a best system, they are not laws since they are not generalisations. However, if one takes laws to be those features of a system which get held fixed in counterfactual reasoning, then it is reasonable to expect some non-generalisations to be counted as law, such as the past hypothesis (Albert, 2015; Jaag \& Loew, 2020). Moreover, its hard to see why the mere syntactic feature of being a generalisation should play a role in what gets held fixed modal robustness is surely more a worldly matter than syntax!

It anyway seems that there will be generalisations in canonically chosen best systems which are implausible candidates for laws, at least so long as the systems permit things like the phony constant or simple summaries. Roberts (2008) and Woodward (2014) discuss the generalisations that would follow if the initial conditions of the solar system were included among Newton's laws as axioms (also see Hicks, 2018). Since the initial conditions will include the planets' velocities, it will be a theorem and generalisation of such a system that the planets all orbit in the same direction. But this is not a law. A similar example discussed by Jaag and Loew (2020), originally from Lange (2009), involves an initial condition specifying the total charge $Q$ of the world which combines with the conservation laws to generate the generalised theorem that the charge of the world is forever and always $Q$. Again, this is not a law.

\footnotetext{
${ }^{5}$ Hall never says how this constant is to be included within the best system. Presumably it can't just sit there as a number and must be incorporated within some statement of the form ' $X$ is a constant which encodes all the coordinates, etc. and can be interpreted according to the rule $R$ '. I think it would be reasonable to complain that the complexity of the interpreting rule $R$ would obviate the inclusion of the statement in a best system, but I put this issue to one side for the present discussion.
} 
In response to these kinds of examples, a point emphasised by Hall and taken up by Jaag \& Loew (2020) is that the laws of fundamental physics as we actually find them have a certain 'modal latitude'. While they comprise strong, informative, dynamic hypotheses, they are broadly accommodating, widely applicable, or 'uninformative' about varying initial conditions. This is evidenced by physics' apparent preference for deterministic laws which permit a wide range of possible values for their parameters. By contrast, the canonical BSA seems liable to attribute the status of law to generalisations which don't have this feature.

As well as attributing lawhood falsely, the canonical BSA has also been charged with missing out genuine laws. One reason for this is its emphasis on simplicity. Woodward (2014) considers a world in which some function $F$ describes a regularity between two quantities $x$ and $y$ in such a complex way that 'we would be unable to comprehend or use it', whereas there is also another true function $G$ that 'relates a summary of $x$ to a summary of $y$ '. Since it is far simpler (by virtue of being easier to process) than $F$, it is liable to be preferred as a law in a canonical best system over $F$ despite being less precise. Yet, as Woodward complains, 'if anything deserves to be called a fundamental law of nature in this scenario, it is surely the regularity described by $F$ rather than the regularity described by $G$ ' (ibid. 29).

A similar example comes from Hicks (2018) who describes 'Taylor's World', in which 'there is a true, informative statement about force that cannot be finitely stated.'

This force statement is a function of a particle's velocity, and acts to damp the motion of the particle:

$$
f_{T}=-a v-b v^{2}-c v^{3}-d v^{4} \ldots
$$

The faster something moves, the more Taylor's force opposes that motion. But each subsequent coefficient $(a ; \ldots ; d ; \ldots)$ is much smaller than the one before it. So at low speeds it can be approximated. (Ibid. 992)

As Hicks remarks, 'because $f_{T}$ has no finite expression, any systematisation that contains it will be maximally unsimple'. Accordingly, any system which includes $f_{T}$ will be no less simple than a mere list of facts and so Taylor's world will be one without laws according to canonical BSA. But as Hicks stipulates (and we may suppose it is plausible), scientists in Taylor's world may be engaged in active research programmes to improve their approximation of Taylor's law, and are able to apply their knowledge to inform action.

A related worry along these lines concerns the tractability of the physical constants. In considering the status of the phony constant in canonical best systems Hall notes that 'it is part of the practice of physics to include in its theories fundamental constants', but 'it is no part of the practice of physics to insist that those constants have mathematically tractable values' $(2015,270)$. Hall's phony constant presents a dilemma for defenders of the canon in that they either have to accept it because of an over-prioritisation of simplicity or else try and reject it on the grounds of intractability and therefore risk throwing out genuine laws with equally intractable constants. Within this there is, therefore, a worry similar to 
that expressed by Hicks and Woodward that an overemphasis on simplicity might lead the canonical BSA to miss some of the laws. Indeed, it is a point which has been repeatedly emphasised that scientists simply do not seem to want to trade off strength for simplicity in anything like the way canonical BSA seems to advise (Roberts, 2008; Woodward, 2014; Hall, 2015; Hicks, 2018).

In response to these kinds of concerns, Hicks and Woodward suggest that simplicity should be given a less weight than strength in the trade-off. Woodward suggests, on the Humean's behalf, that simplicity considerations should come into play only for systems whose strength have reached a certain threshold (2014, 101). However, Hicks provides replacement desiderata for the canonical notion of strength which include a preference for the laws having as instances a greater quantity of ('breadth'), and greater detail ('local strength') about, quasi-isolated and approximate quasi-isolated systems, i.e. instances whose variable properties fill the laws' parameters while preserving their truth or approximate truth (see esp., ibid. 998-1001). Hicks also recommends a preference for laws which are appropriately modular (in the sense of being independently testable) and simpler (in the sense of having fewer free parameters) but suggests these desiderata should have 'less weight' than the others.

Much of the motivation behind Hicks's specific substitute desiderata come from the observation that the canonical conception of strength is too 'global' (see also Jaag \& Loew, 2020). Hicks remarks, for example, that 'the information provided by a globally strong lawbook may only allow agents to make predictions if they know just where in the world they are; but agents are often operating with very limited knowledge about their position in space or time' (ibid. 998-9). Consequently, Hicks argues, 'we should seek a lawbook that can be applied by agents with little or no global knowledge'.

Dorst expresses the similar worry that the canonical desiderata do not make sufficient reference to the actual features of scientific activity and instead aim for a 'God's-eye-efficient summary' of the world.

It's possible that the comparatively useless information about distant events will swamp out the more useful information about how things behave around here [...] Alternatively, it's possible that the information we care about won't get swamped out, but instead it will be condensed into a form that makes it unusable. (Dorst, 2019, 885)

Dorst's criticism effectively takes issue with the appropriateness of the analogy mentioned in passing by Lewis of the laws being those generalisations God would put into an encyclopaedia for us of the history of the world in order to make it as concise as possible. Dorst's point is that the needs of God (being omnipresent) are not our own, and it would be wrong to pin what is characteristic of a law to whatever generalisations God would consider efficient. Using the metaphor of navigating a maze he argues that it would be preferable to have information about which direction to take given one's position than, say, the total number of lefts and rights needed.

To remedy, Dorst offers an alternative 'best predictive system account' according to which the laws are generalisations of the system that achieves the best balance of 
revised desiderata which include maximally informative dynamics (ibid., 887), high generality and permissiveness with respect to initial conditions (ibid. 888), spatial locality (ibid. 889-90), temporal locality (i.e. Markovian dynamics; ibid. 892), translational, temporal and rotational symmetry (ibid. 893) and describing relations between properties which are independently ascertainable (ibid. 894-95). A system chosen for its balance of at least the above desiderata would, Dorst argues, be far more likely than the canonical formula to output generalisations 'sufficiently pragmatic to be worth our attention' (ibid. 884).

Despite different emphases (respectively on epistemic access and practical action) both Hicks and Dorst's strategy is to respond to the canon's counterexamples by replacing the canonical desiderata with more pragmatically inspired desiderata. As they both acknowledge, this idea is already present in Hall, who surmised that the canonical BSA emphasises the 'the wrong kind of' strength (ibid. 270). Hall's own suggestion (e.g. ibid. 268) is that properly conceived, strength should have something to do us, with our idiosyncratic goals and cognitive and physical abilities, which we find helpful to know for the sake of prediction, explanation and action-guidance. ${ }^{6}$

Jaag \& Loew (2020) also explicitly take up Hall's idea, suggesting that strength should somehow be tailored to the fact that we are finite beings typically with partial, incomplete and often imprecise information of the world. We are also, they note, limited by our ability to process information and by the time and resources we have to gather and store the information necessary to get perfectly accurate predictions. It is therefore appropriate that our laws have a high degree of 'error-tolerance' (ibid. 2535) and permit successful predictions using only 'partial models' (ibid. 2537). Whatever the desideratum of strength amounts to, they argue, it needs to be one which prioritises these features. They consequently offer an alternative desideratum aimed at capturing these features in terms of a preference for systems which maximise 'cognitive utility' (ibid. 2533).

Summing up, the case against canonical BSA seems strong. Apparently it neither accommodates all the laws nor does it block all the non-laws. The critics we have considered propose, though in different ways, the same strategy for solution: revise the canonical desiderata of simplicity and (global) strength by adding in new more pragmatically inspired ones. The hope seems to be that in doing so, the best systems are more likely to give us the laws. In what remains, I'll grant that all the proposed revisionary desiderata are represented to some extent by the world's actual best system (or systems, see below), though not perfectly, as we will see. ${ }^{7}$ Nevertheless, my aim over the next two sections is to show that the revisionist strategy is both unnecessary and inappropriate.

\footnotetext{
${ }^{6}$ Elsewhere, Hicks has suggested that we should think of embedded agents in terms of Hartle's 'information gathering and utilising systems' (Hartle, 2005).

7 My only serious doubts concern Dorst's final desideratum. I can't see why it would even be pragmatically useful for laws in the actual world. Obviously we can't use unknown properties to predict others, but we also don't care much about predicting properties via laws which are easy to determine by other means (except when testing the laws themselves). Laws are useful insofar as they concern both properties we can determine without the laws and properties we can't, or can't very easily.
} 


\section{The canon defended}

The foregoing issues draw inspiration on the pragmatic turn in order to suggest revision to the desiderata for best systems. But before assessing these revisionary accounts on their own merits we should pause to consider whether revision is really necessary. The Humean can, I take it, coherently accept the reasoning behind the pragmatic turn while retaining a preference for the canonical desiderata only. And in fact Humeans have a number of choice points and facts in their favour when it comes to thinking about the canonical desiderata which offer scope for defence against the issues just identified within canonical constraints.

One choice point concerns how the Humean should understand the notion of simplicity. We saw earlier how Lewis likened comparisons of simplicity to that between a linear and quartic function (Lewis, 1994, cf. Hicks's definition in terms of quantity of free parameters). This can make it seem that irrational constants, complex functions like Woodward's $F$, or Hicks's Taylor's law could never get into the best system. $^{8}$ But I think this likely trades in a limited understanding of simplicity. 'It can scarcely be denied,' Einstein once remarked, 'that the supreme goal of all theory is to make the irreducible basic elements as simple and as few as possible without having to surrender the adequate representation of a single datum of experience. (Einstein, 1934, 165, my emphasis). Correspondingly, the defender of canonical BSA should think the relevant understanding of simplicity must take into account the simplicity of the system by virtue having fewer non-conjunctive axioms. Despite its lack of finite expression Taylor's law is stipulated to be just one axiom describing one kind of behaviour (hence non-conjunctive). With other suitably deterministic laws (e.g. a total force law) and thoroughly specified initial conditions the entire history of a whole world may be derived. That appears to be a lot simpler, in this alternative sense, than a set of axioms comprising a long list of historical events. If the desideratum of simplicity can, in line with Einstein's claim, take into account the quantity of non-conjunctive axioms then worries about whether or not laws with irrational constants or complex laws like Taylor's law can be included may not arise.

But the defender of the canon should also query the extent of the putatively unsimple laws. For it is surely implausible for the Humean that we reject the relevance of syntactical simplicity altogether. Woodward talks of 'some function $F$ ' able to describe a regularity between two quantities $x$ and $y$. Yet in a finite world there can be functions for any otherwise seemingly random sequence no matter how long. So there must be some threshold to the permitted complexity of functions such that when they surpass it they are no longer plausible candidates for laws. Indeed, presumably this is what the Humean must say of quantum indeterminacy if fundamental indeterminacy is to be retained in the laws. Although there might be 'some function' which determines exactly how waves will collapse in all actual cases, its complexity will not be mitigated by its strength.

\footnotetext{
${ }^{8}$ It's anyway not obvious that the revisionary approaches solve the issue identified by these imagined laws. If simplicity has an influence on the overall scoring independently of the scores of other desiderata, as Hicks and Dorst seem to allow, then such laws may still be denied from the BSA whatever other revisionary desiderata we add in.
} 
So while the Humean may be able to accommodate more complex laws than some have thought (e.g. Hicks's Taylor's force law), they should also resist the pressure to accommodate laws of any non-conjunctive complexity. While Taylor's law may be infinitely long, it has a predictable syntactic form. Other functions will be less elegant and predictable in structure. For these, Humeans should permit syntactic complexity to count against them. Ultimately, then, the defender of the canonical BSA should advise that simplicity itself be understood, in line with Einstein's own observation of the goal of theory building, as a balance between syntactical simplicity and the simplicity afforded by having fewer axioms.

The interpretation of simplicity just offered will not address all the problems facing the canonical BSA. In particular it won't help address the issue of the phony constant and potential exact summaries, which may be somewhat complex in length but are non-conjunctive and would presumably count as a single axiom. It's worth emphasising, however, that adding a phony constant or exact summary into a system is only conducive to a significant increase in strength if the dynamical laws they sit alongside are deterministic. It could be, however, that a far simpler system is achieved which compromises on strength by sacrificing determinism. As was just pointed out, if certain popular interpretations of quantum mechanics are anything to go by this seems exactly what the Humean should say is the case for our world. But in that case, adding a phony constant or indeed any kind of exact summary alongside indeterministic generalisations will not help increase strength much, since nothing much will be determined from them. Notice, by the way, that this provides a response to the charge that strength is not in practice compromised for the sake of simplicity (Roberts, 2008; Woodward, 2014): not only can simplicity considerations justify a preference for a significantly less strong system, this is plausibly the case for our actual physical laws!

What if deterministic generalisations can be achieved fairly concisely though? In that case we can't preclude phony constants or exact summaries on the grounds that they won't increase strength much. But the defender of the canon still has options. Another two relevant and crucially distinct choicepoints concern what gets called a law in best systems and what gets held fixed in counterfactual reasoning. It seems to me exactly right to respond to the concern that Hall's phony constant and exact summary will appear in the best system in the way suggested earlier: a phony constant or exact summary may have a place in a system, but since they aren't generalisations, they aren't laws. ${ }^{9}$ Historically, the concern with this response has been that it either requires that the phony constant/exact summary be held fixed when reasoning counterfactually (because they're in a best system) or else preclude the past hypothesis from being held fixed (because its not a generalisation). The trick is to justify why

\footnotetext{
9 It has been objected that some of our best candidate laws, e.g. Newton's second law and the Schrödinger equation, are not regularities/generalisations (Maudlin, 2007; Jaag \& Loew, 2020). See Friend (2016) for a reply.
} 
we hold fixed the latter but not the former. More generally, we want a principled reason to hold fixed only a subset of the theorems of the best system where that subset includes more than just the theorems which are generalisations. For instance, in the case of the actual world, that subset should also (if Albert is right) include the past hypothesis. ${ }^{10}$

I suspect a good place to start is by noting that any phony constant or exact summary could just as well be replaced by an equally complex statement of the state of the world at some other time (or along a different hyper-surface) whereas the past hypothesis most certainly could not be (it's essentially about the distant past). ${ }^{11}$ In effect, the thought is to acknowledge that many systems (potentially individuated by the inclusion of different phony constants or exact summaries) may in fact achieve a best combination of simplicity and strength. This was anyway something both Lewis and Ramsey were open to. ${ }^{12}$ To be a law of a world, they thought-and the defender of the canonical BSA should agree-a proposition must feature as a generalisation in all the bestachieving systems. For our world, the past hypothesis also features (we think) in all of those systems too, and that's justification enough for holding it fixed along with the systems' generalisations. But since any particular phony constant or summary features in only a subset of the best-achieving systems, it isn't held fixed.

If we allow phony constants and exact summaries in the best systems, what else might sneak in? Well, whatever is conducive to helping the system succeed in being best-achieving in simplicity and strength. At least, we needn't worry that letting in phony constants and exact summaries will open the doors to any old junk. Moreover, if we can hold things fixed (because they're in all the best systems) without having to call them laws, then lawhood can remain a concept safely restricted to generalisations only. There will no longer be the worry that what gets held fixed is just a matter of syntax. I suggest furthermore that the Humean restrict laws only to generalisations which are theorems of whatever gets held fixed (i.e. the theorems of all best systems). This is close to what Ramsey had in mind initially when he claimed that the general axioms in the best system(s) would be the 'fundamental' or 'ultimate' laws of nature, and that derivative laws would be 'general propositions deducible from the ultimate laws' (Ramsey, 1928, 130-1). If this further restriction is maintained then the theorems of the best-achieving systems which we call laws will not be tainted by initial conditions or other matters of particular fact (besides the past hypothesis) and

\footnotetext{
10 According to Albert (2015) we should also hold fixed the statistical postulate that specifies a uniform probability distribution over the microstates compatible with the past hypothesis. Albert argues this and the past hypothesis must be laws by virtue of their modal role; I'm suggesting here that they can both play that role without having to be called a law.

11 Ramsey initially conceded that 'the choice of axioms is bound to some extent to be arbitrary, but what is less likely to be arbitrary if any simplicity is to be preserved is a body of fundamental generalisations' (ibid. 131). I'm suggesting that the past hypothesis isn't so arbitrary either.

${ }^{12}$ Lewis claimed that laws are the contingent generalisations which appear in 'each of the true deductive systems which achieves a best combination of simplicity and strength' (Lewis, 1973, 73, my emphasis) (see also footnote 11).
} 
consequently, generalisations about the matching angular velocities of the planets or the total value of charge for all time will not get counted as laws. ${ }^{13}$

Manoeuvres like these show the versatility of the canonical BSA thesis in accommodating many of the putative counterexamples presented in Section 4. Nevertheless, no doubt there will be some lingering concern from the revisionist camp. Even if simplicity and strength can alone render the laws practically useful for us, the worry might remain that they are unlikely to do so. For instance, Dorst claims that the potential for the canonical desiderata to provide Markovian laws 'would seem to be merely a stroke of luck-a convenient accident as it were' (Dorst, 2019, 892; where similar remarks could be made for other desired characteristics, see his fn.17).

The complaint that the best systems will be lucky to get the laws right is ambiguous between the claim that the canonically chosen systems fail to be robustly likely to ensure the laws actually exhibit the pragmatic virtues praised by revisionists (e.g. Markovian dynamics) and the claim that the canonical systems fail to be robustly likely to ensure the laws exhibit the pragmatic virtues in all worlds. ${ }^{14}$

If the complaint is the former, then it really shouldn't concern the defender of the canon much. If the canonical best-achieving systems do happen to get the laws right in the actual world then they necessarily get the laws right in the actual world, since there is only one actual world and by stipulation the canonical systems get the laws right in it. Nor can it be objected that while the canonical formula might in fact output the actual world's laws a revised formula more tailored to the laws' pragmatic characteristics is more robustly likely to output them. For the Humean who follows Lewis's (1986) priorities for counterfactual reasoning, whether something contingent holds robustly under counterfactual supposition is first and foremostly a matter of what the actual laws (alongside whatever else appears in all the best-achieving canonical systems) are. The laws of the actual world determine, along with contingent matters of fact, which worlds are those whose ongoings are relevant to the truth of counterfactuals and hence what consequent conditions are robust. So if the canonical desiderata do in fact manage to identify the law-giving systems of the actual world then they do so as robustly as any set of desiderata.

\footnotetext{
${ }^{13}$ Is it fair to pick out laws only by this syntactic and deductive feature? A worry remains that since any axiom $a$ can be put in the form of a generalisation, e.g. $\forall x((x=x) \rightarrow a)$, then all modally robust axioms will be laws (thanks to John Roberts for pressing this objection). The Humean will therefore likely require some constraint beyond syntax. On this issue I can offer only some less than fully satisfactory remarks. First, I suggest that it is here where compact expression counts, not as a desideratum for choosing the best systems but as a method for deciding on how they should be expressed and picking the laws from them. I suggest that generalisations be allowed to count as laws only if there is no simpler way to express them in a different form. Clearly $\forall x((x=x) \rightarrow a)$ can be more simply expressed, as $a$, so it doesn't count as a law. Beyond this, the Humean may also want to restrict generalisations to the form $\forall x(S x \rightarrow B x)$ specifically where $S$ picks out some natural kind of system and $B$ some behavioural property in terms of the functional relationships among the relevant systems' variable properties. I have argued elsewhere that laws should have this form (Friend, 2016).

${ }^{14}$ Under the first disambiguation, given the actual world, the canonical systems are unlikely to be Markovian (etc.). Under the second, a measure over all the worlds would return a higher or equal density for worlds in which the canonical systems have non-Markovian (etc.) dynamics than Markovian (etc.) dynamics.
} 
If, however, the complaint is that the canonical systems fail to be robustly likely to ensure the laws exhibit certain pragmatic virtues for all worlds then the defender of the canon must agree. There is nothing in the competition for the canonical best systems alone which ensures that the laws of any world will tend to be Markovian, local, exhibit the symmetries, permit error-tolerance and partial modelling, be tractable, have wide applicability and informative dynamics. The question is whether the competition for best systems should do this. In the next section I try to show why we have reason to doubt it that it should.

\section{Pragmatic how?}

As we've seen, the revisionary desiderata are justified on a number of varying pragmatic grounds. To help organise a comprehensive discussion of them I suggest we draw on Demarest's (How (Not) to be a Pragmatic Humean, unpublished MS) useful distinction of three ways in which the laws are useful.

(i) Laws help us get what we want.

(ii) Laws only require information to which we have access.

(iii) Laws are easy to use.

Revisionists have motivated their revisionary desiderata in terms of each of these ways. However, by dividing up the discussion roughly along these divisions I'll show that doubt can therefore be raised over whether the revisionary desiderata really are justified. As a rule, either the canonical desiderata of simplicity and strength seem to do just fine prioritising the systems which best exemplify these pragmatic virtues or else these virtues are not reasonable features to desire from physical theory, even if physical theory happens to exhibit them.

\subsection{What do we want from laws?}

Let's turn first to the idea that laws help us get what we want. If this is the way Humeans under the pragmatic turn have in mind for how laws are pragmatic it is surely not in anything like the sense of enabling us to get material goods or benefits. Certainly our knowledge of laws have been put to such ends in technological application, but it is unlikely that physics has been driven by such applications. ${ }^{15} \mathrm{~A}$ different sort of thing we might want is information and Humeans engaged in the debate about laws' pragmatic value have by and large been explicit that laws can help us get information by being predictive.

\footnotetext{
15 Arguably, both relativistic theories were developed and motivated on purely theoretical grounds and later confirmed only by highly contrived experiments with no utility in mind. Perhaps a case for pragmatic motivation can be made for quantum mechanics, since the initial curiosities concerning black-body radiation and light-absorption in gases were closely tied to the development of thermodynamics and statistical mechanics (scientific enterprises closely aligned with industry). But the industry and phenomenon-specific issues which are relevant here are a world away from the general agent-based considerations behind the revisionist programme.
} 
We assume that the main cognitive function of the laws is facilitating predictions. (Jaag \& Loew, 2020, 2534)

If I am right that reductionists should view the laws pragmatically, and that the primary pragmatic use of laws is predictive. (Dorst, 2019, 886)

If predictive power is what we want from laws then one might be forgiven for thinking that it is laws' balance of strength and simplicity that is precisely what makes them so helpful. That is, the canonical desiderata seem conducive to this aspect of laws' pragmatic value. This is reflected in what physicists say about what they are looking for. For instance, Einstein remarked that 'the supreme task of the physicist is to arrive at those universal elementary laws from which the cosmos can be built up by pure deduction' (Einstein, 2002). In a more poetic vein, Feynman notes that 'nature uses only the longest threads to weave her patterns, so each small piece of her fabric reveals the organization of the entire tapestry' (Feynman, 1967, 34).

It's long been understood why the canonical desiderata play such a useful role in prediction. To explain Humeans often invoke the parable (initially in Lewis, 1973) of God's compression of the world's history.

Suppose God wanted us to learn all the facts there are to be learned [...] He decides to give us a book-God's Big Book of Facts - so that we might come to learn its contents and thereby learn every particular matter of fact there is. [...] As a first draft, God just lists all the particular matters of fact there are. Luckily, however (or so we hope), God has a way of making the list rather more comprehensible to our feeble, finite minds: he can axiomatize the list. That is, he can write down some universal generalizations with the help of which we can derive some elements of the list from others. This will have the benefit of making God's Big Book of Facts a good deal shorter and also a good deal easier to get our rather limited brains around. (Beebee, 2000, 574)

God's more comprehensible 'Big Book of Facts' would describe the canonical best system, and the universal generalisations contained within it would be the laws. The parable highlights why strength and simplicity are suitable desiderata for axiomatised systems which target generalisations which give us information about the world that we might want, since their regularities will be the kinds of thing knowledge of which massively increase our ability to predict the world given fairly minimal knowledge.

Of course, the key observation of the revisionist programme is that the canonical desiderata are nevertheless somehow pragmatically insufficient. And it's not implausible that what at least some revisionists have in mind here is that the kind of information a canonical competition for best systems prioritises is not liable to facilitate is specifically practical action. Dorst's example (ibid. 882) of what information we would desire when navigating a maze seems particularly indicative of this idea (see also Jaag \& Loew, 2020, 2534) and is in the spirit of Beebee's own remark (ibid. 547) that the information content provided by best systems should be enough for 'making your way about in the world'.

Ultimately, however, it's hard to understand science, especially physics, as being motivated in this way. Demarest draws attention to this in the following. 
There seems to be vanishingly small practical benefits to systematizing patterns that are spatiotemporally distant from us and our day-to-day goals. Think of standard engineering projects: building faster internet networks, or electric semi-trucks, or medicine-delivery nanobots. It is difficult to see why, from this kind of pragmatic perspective, we should bother systematizing patterns in the movement of distant galaxies. [...] If we care about these things intrinsically or because we seek understanding, they are not pragmatic goals and have nothing to do with pragmatic usefulness. [...] Consider the staggering financial resources and research hours that have gone into the Laser Interferometer Gravitational-Wave Observatory (LIGO). LIGO has cost over a billion dollars, with hundreds of scientists contributing to its research projects. Its purpose is to directly detect gravitational waves. Yet, if those resources had gone instead towards laws that systematize only the spatiotemporally local environment on Earth, plausibly, the result would be more useful laws - at least insofar as usefulness is about getting what we want or making our way about. (Ibid. 6-7)

The central point Demarest wants to make with these remarks is that the canonical (or 'Lewisian') BSA is not well-adapted to being conducive towards the kind of pragmatic goals revisionists would have to seem to want if they think the laws should be conducive to action-guidance. But these remarks also reveal how implausible it is that physics is really concerned with practical action. Physics is precisely concerned with things like the movement of distant galaxies and whether or not there are gravity waves as opposed to being concerned with how to more effectively perform our day-to-day goals. Demarest admits herself that she is 'skeptical that pragmatic usefulness should play such a large role in justifying our pursuit of the laws of nature. [...] Indeed, I think some of our paradigmatically excellent examples of scientific inquiry are best characterized as "pure research," appropriately unconcerned with potential practical applications' (ibid. fn.10).

The view is not unfamiliar. Consider the following description of the aims of physics from Davidson.

We don't know, and for practical purposes don't care, what would happen if there were no oxygen, temperatures were to fall to absolute zero, or there were a black hole in the closet. [...] The more precise and general laws are, the less likely it is that we will be in a position to employ them in predicting the outcomes of our ordinary actions or the weather. [But] we have interests that are not practical. There are things we want to understand whether or not we can control them and whether or not such knowledge will serve our mundane needs. Pursuit of the truth in such cases can in principle proceed without the constraints of practical control and gain. In this mood we can seek laws that have no exceptions. (Davidson, 1995, 275-76)

This understanding of the goals of physics enquiry doesn't preclude there being significant action-guiding practical benefits to engaging in physics. But the explanation of how these benefits come about seems to be rather that they are by-products of the enquiry rather than constitutive of it. However, if it's wrong to think of the kind of constitutive practical value laws have as being one of facilitating action-guidance then it can't be a failure to ensure 
such information that is ultimately what is wrong with the canonical BSA. Indeed, the predictive generalisations physics seems to be aimed at getting us is exactly what canonical strength and simplicity seem apt to single out, in the sense of facilitating an efficient representation from which the cosmos can be 'built up by pure deduction'.

This does not entail that the laws of physics are theorems of the canonical best systems, of course. One reason they wouldn't be is if the notions of strength and simplicity are not specific enough to give us the kinds of predictions we want. Revisionsts seem to suggest this when they emphasise the requirement that generalisations have applicability to a wide range of systems whilst retaining informative ('locally strong', in Hicks's sense) dynamics. Dorst argues these are things we want because we want to make predictions about how many different token systems will evolve over time.

It is surely a highly reasonable observation about our best theories of fundamental physics that they seem to carve out the nomologically possible worlds by having fairly weak constraints on initial conditions but strong constraints on dynamics (see especially Hall, 2015, but also Woodward, 2014; Dorst, 2019; Jaag \& Loew, 2020). Nevertheless, I doubt that the genuinely desirable aspects of these features of laws add more relevant specificity to the canonical desiderata of strength and simplicity (understood in the terms suggested in Section 5 in terms of few, simply formulated non-conjunctive axioms). One way of saying a lot while remaining minimal in number just is to apply to a large number of instances. And if those instances differ from each other in various ways then, as Dorst (ibid. 888) himself points out, the generalisations will have to be sensitive to the differences among these systems. Moreover, if some of these differences can be captured by a quantitative scale of a single variable property then it's likely that wide applicability will be exemplified numerically. Moreover, since locations in time and space are essentially just further differences systems can exhibit, then the notion of applying to wide range of systems extends readily to being informative about dynamics too. ${ }^{16}$

In the end, it doesn't seem like wide applicability and informative dynamics are importantly distinct from the basic criterion that laws say a lot while remaining minimal in number and simply formulated, as per the foregoing characterisation of simplicity for the canonical BSA. That laws exhibit these features cannot therefore be used to undermine the canonical BSA. In this way, the complaint that canonical systems will fail to have informative dynamics or concern many physical systems is no better than attempts to show that canonically chosen generalisations will fail to get us material goods or facilitate practical action, though the complaints suffer in different ways. The latter complaint fails because laws just don't seem to be the sorts of things which are supposed to provide us with these things, the former fails because the canonical desiderata already explains why they would.

\footnotetext{
16 Of course, dynamics tends to by understood as concerning the evolution of a single physical system at different times and spatial configurations, rather than a range of different system-stages varying along a continuum. But the identity of systems are themselves defined by the laws, e.g. regions which are materially conserved. So the specificness of dynamical generalisations attending to a single system shouldn't be overemphasised in the context of saying what characteristics are constitutive of laws.
} 
However, a rather different reason to think the laws of physics could not be theorems of the canonical best systems is that there may be other practical virtues of the laws beyond getting us what we want. It is here where Demarest's other ways laws are useful come into play.

\subsection{What can we access?}

That epistemic accessibility is a key constitutive feature of laws' practical value is suggested by Dorst's (ibid. 892) observations that 'spatial and temporal locality desiderata arise because we do not typically have information about spatially and temporally distant events' and that 'we often do not have is information that allows us to locate and orient ourselves within space and time' (there are similar remarks from Dorst about utility of Markovian dynamics and ascertainable magnitudes). Hicks (2018) is even more explicit about accessibility being a constitutive feature of laws.

Scientists are looking for generalizations that can be known by observing a subset of their instances in controlled situations. (Ibid. 994)

Science seeks to extend our knowledge from those contexts wherein we gain evidence to those in which we make predictions. Scientific laws have a central role to play in this extension. (Ibid. 996)

It is for these reasons that Hicks suggests his further criteria of a maximum quantity ('breadth') and greater detail ('local strength') concerning quasi-isolated and approximate quasi-isolated systems and a (weaker) preference for laws to be appropriately modular and have fewer parameters. Each of these criteria, like those mentioned by Dorst, are supposed to reflect the kind of information that we in fact have access to, and therefore which kinds of information generalisations of the best systems should tend to have as theorems.

There is an issue, however, with at least some of these desiderata being justified in this way. Revisionists have tended to develop their accounts treating the limitations of any creature like us to be akin to our limitations in this world. For instance, Hicks remarks that 'agents are often operating with very limited knowledge about their position in space or time' (ibid. 998-9) and so 'laws must be discovered empirically by limited agents operating locally' (ibid. 984). His proposed desiderata therefore aims to reflect the kinds of information which is actually epistemically accessible. But in general there doesn't seem any good Humean reason to suppose that empirical enquiry is necessarily constrained by the sorts of inductive abilities revisionists have tended to prioritise.

For instance, it seems entirely possible that some worlds could admit rife and systematic non-locality (at least beyond agents themselves). Granting that, it's hard to see why agents in some such world should not therefore be able to sense directly the ongoings of distant occurrences. As Hicks and Demarest have elsewhere remarked in co-authorship, 'tools to measure nonlocal correlations could themselves be nonlocal' (Demarest \& Hicks, 2020, 10). Indeed, the very reason we can't make such observations or develop such tools in the actual world seems to be exactly because our (macro) laws happen to be local: if the laws had been different, so would our 
abilities. In a non-local world, however, a preference for locality would no longer be a desideratum which would tend to prioritise systems more epistemically accessible. From the Humean perspective, there's nothing abnormal about such a world. Some worlds have rife local correlations, some (most?) don't. But if that's all correct, then a preference for local generalisations cannot be a constitutive desideratum for the best systems only on the grounds that it tends to prioritise more epistemically accessible systems, since the desideratum's tendency to do so is at best entirely dependent on contingent features of the way the world is. Indeed, such a preference may just as easily be antagonistic to prioritising a more accessible system, since in some worlds information gleaned only from prioritising contrary desiderata (e.g. non-locality) will in fact be more (or just as) easily accessed.

This last observation blocks a possible response on behalf of the proposal of locality on grounds of access. The revisionist making such a proposal might admit worlds where non-locality is preferred among the best systems but suggest their laws' non-locality can be put down to the fact that locality is permitted to take a low score because of superlative scores on other desiderata. If that were the correct assessment of such worlds then we could say of them that it would be preferable if their laws exhibited more locality at no cost to other desiderata. But that is just what is not the case. It is non-local generalisations which are praiseworthy for their epistemic accessibility in these worlds. Local information is therefore not even desirable in an of itself.

Notice that it's also no use for the revisionist who wants to propose locality as a desideratum on the basis of its conduciveness to epistemic accessibility to permit its status as a desideratum (or the specific weights apportioned to it) to be worlddependent, e.g. applying only to our world. It is the presumably the job, or part of the job, of a philosophical account of laws to say what it is to be a law of nature. At least, that's what Humeans have always been up to in the past. Hence, the account should say what it takes to be a law of nature in any arbitrarily chosen world. If some desideratum (or its weighting) is world-dependent then it can be no assistance in this kind of project and so should not be considered a genuine 'desideratum' at all.

The issue just described plagues other suggested desiderata too. A world might conceivably be non-Markovian in the sense that agents my be able to glean information directly about ongoings in the distant past or future. Correspondingly, tools to measure correlations could themselves be non-Markovian. Alternatively, if the world were more as Aristotle conceived it, with a privileged centre to the universe, our inductive capacities would presumably have trained us to tell where we were oriented in relation to the centre of the universe by looking at how things behaved around us. Indeed, until the Copernican revolution, many thought this was exactly what we were doing! But in that case, our epistemic access wouldn't satisfy all the symmetries which they do in the actual world. A boost in some direction or a reorientation might be plainly obvious to agents by virtue of the differences in how things are moving in relation to them. Consequently, and as with spatial localities, a preference for all the symmetries or Markovian dynamics are not desiderata which would tend to prioritise systems more epistemically accessible in some worlds. Indeed, such preferences, like the preference for rotational symmetry in a world in which the most widespread correlations exhibit a failure of rotational symmetry, may in fact be antagonistic to prioritising a more accessible system; in some worlds information 
gleaned only from emphasising contrary desiderata (e.g. reflecting rotational asymmetries) will in fact be more easily accessed. Such preferences cannot, therefore, be the constitutive desiderata for prioritising systems on the grounds that they tend to promote epistemically accessible systems. ${ }^{17}$

These problems are enough, I think, to undermine the employment of certain desiderata on the grounds of their provision of more epistemically accessible systems. Specifically, they undermine the preference for locality, Markovian dynamics and rotational symmetry on such grounds. I take it that analogous arguments can also be provided for other global external symmetries proffered by Dorst as desiderata (i.e. spatial and temporal symmetries). Moreover, as I'll end this subsection by showing, the failure of all these desiderata in this regard highlights once again the power of the canonical desiderata.

First notice that the fact some desideratum D is only contingently beneficial to prioritising systems whose theorems involve information which is epistemically accessible in a world raises a question over why it is beneficial in some worlds. And here, I take it to be entirely natural to claim that this will have something to do with what the laws are in that world. For example, it is because the actual laws are by and large ones which chart local interactions that local information actually tends to be more epistemically accessible than non-local information. After all, what else could be meant when we considered (over the preceding paragraphs) worlds in which measurement and tools are 'non-local' or in which there is a privileged centre, than that the laws enable such measurement or establish such a centre?

The claim that it is due to the laws that D is contingently beneficial or antagonistic to prioritising epistemically accessible systems is, of course, entirely inconsistent with the proposal that it is constitutive of laws that they be those theorems of a system chosen for its best-achieving balance of desiderata which include D on the grounds that D is beneficial to prioritising epistemically accessible systems. If the laws explain why D is conducive to epistemically accessibility then D can't also explain why the laws are epistemically accessible. (For comparison, a Humean wouldn't say that it is because the best system's generalisation permit such comprehensive predictions that strength is constitutive of them; rather, it is because strength is a constitutive desideratum that the laws tend to permit such comprehensive predictions.) So we will have to look elsewhere for an explanation of epistemic accessibility, and for the Humean especially, the canonical desiderata are obvious candidates.

Of course, the revisionist who wishes to uphold the constitutive nature of D may just reject the intuition that the laws have this explanatory role. It is harder, however, to reject the observation that we can imagine worlds where agents have robust epistemic access to non-local, non-Markovian generalisations or generalisations which

\footnotetext{
17 There seems anyway to be a strong case for global external symmetries not being characteristics of our theories which it is the duty of the competition for best systems to uncover. The idea that the explanatory power of symmetries comes merely from being theorems of the best systems has come under serious criticism (Lange, 2007, 2011; Hicks, 2018; Friend, forthcoming). According to all these opposing accounts, symmetries are to be sought elsewhere. Being symmetrical should not, therefore, be considered a desideratum for finding the best system.
} 
reveal rotational, etc. asymmetries. And once we grant that there are such worlds we can enquire about what it is exactly we are imagining when we think of them. What we are imagining, I suggest, are worlds where the most comprehensive regularities are simply described in terms of non-local, non-Markovian and asymmetric correlations. But this strongly suggests that it is the generalisations which follow from the canonically-chosen best systems which determine whether certain correlations are epistemically accessible in a world, and if that's the case, then additional desiderata introduced to prioritise generalisations about those correlations are clearly redundant for finding the laws which are epistemically accessible.

The forgoing observations once again lend support to the canonical desiderata while presenting significant challenges to the promotion of locality, Markovian dynamics and symmetries as revisionary desiderata on grounds of epistemic accessibility. But these are only some of the revisionary desiderata proposed on such grounds. Specifically, it is less obvious that Hicks's revisionary desiderata can be undermined in the same way. Arguably, the independent testability (modularity) and breadth (in quasi-isolated instances) of a system's generalisations could never be antagonistic to its epistemic accessibility in the way just discussed. ${ }^{18}$ Nevertheless, I do not think these are particularly plausible desiderata either. I'll return later to consider their status in the next subsection once we have considered motivations based on ease of use.

\subsection{Does physics care?}

The third of Demarest's ways in which laws may be pragmatic is that they are easy to use. The Big Book of Facts metaphor has also been presented in order to stress this. Beebee $(2000,547)$ suggests that the information in the Big Book would preferably be 'comprehensible to our feeble, finite minds', and revisionists have picked up on this idea. Hall $(2015,268)$ talks of our 'needs and limitations as inquirers' and implies that mathematical describability should play a role in assessing whether, for instance, the claim that there are exactly $2^{64}$ particles in our world should count as a law (ibid. 269). Jaag and Loew take a similar view.

A system that is best for us should facilitate solving as many of these problems as possible with as little cognitive effort as possible. Such a system has to allow us to make the relevant predictions from the available input without being too demanding on our capabilities for storing and processing information. (2020, 2534; see also their examples on the same page.)

When asking what features of a system could make it easy to use (for our feeble, finite minds) the canonical desideratum of simplicity seems like it should be an obvious candidate. Moreover, simplicity is a feature noted both of laws as we find them and what we aim to achieve in physics. Recall the earlier claim of Einstein's

\footnotetext{
18 The desiderata might score lower for the winning systems than for some other systems, but one can still say that it would be preferable if the winning systems had scored more highly holding fixed the other scores.
} 
that the 'supreme goal of all theory' involves making 'the irreducible basic elements as simple and as few as possible'. And it has been observed that physicists have historically been quite successful on this count.

[T] he most impressive fact is that gravity is simple. It is simple in its pattern.

This is common to all our laws; they all turn out to be simple things, although complex in their actual actions. (Feynman, 1967, 33)

But if revision to the desiderata for best systems is to be motivated by laws' ease of use then simplicity can't be all there is to it. Jaag and Loew proffer a prioritisation of systems which also facilitate partial modelling and error tolerance as further desiderata. Alongside these we might also suggest computational tractability. Like Hicks's desiderata of modularity and breadth — and unlike Dorst's desiderata of locality, Markovian dynamics and symmetry-it is hard to imagine worlds in which these desiderata would not be conducive to prioritising systems which exhibit the grounds which motivate them. More breadth, modularity, partial modelling, errortolerance and tractability can seem to be always worth pushing for however the world turns out.

Even so, that doesn't mean these desiderata are the right ones for the laws. Indeed, some of these desiderata seem to be in conflict with other claims made with which some revisionists have expressed sympathy. Consider again Woodward's example of the function $F$ (alternatively, Hicks's example of the Taylor force law). Woodward's intuition is that there could be a world in which some function $F$ describes a regularity between two quantities $x$ and $y$ in such a complex way that 'we would be unable to comprehend or use it', to which we might add that it fails to be computationally tractable, not employable for partial modelling and completely error intolerant. We may also add that $F$ only applies to the universe as a whole and that it can only be tested in conjunction with some other equally precise function $H$, so that it is not at all broad or modular in Hicks's sense. The function $F$ is then to be contrasted with some other function $G$ which we may suggest scores better on some or all of these desiderata but only manages to 'relate a summary of $x$ and $y$ '. Along with Woodward's original intuition (analogously Hicks's for the Taylor force law) we should plausibly surmise that if anything deserves to be called a fundamental law of nature in this scenario, it is surely the regularity described by $F$ rather than the regularity described by $G$ '.

The generalised Woodwardian intuition here appears to go directly against the thought that considerations of usability are relevant to lawhood. Of course, I argued above that Woodward's argument is not a good argument against the desideratum of simplicity specifically if we take simplicity to be partly a matter of syntax and partly a matter of quantity of non-conjunctive axioms. Moreover, I suggested that the Woodwardian intuition shouldn't go unrestricted. There is always some non-trivial threshold of complexity (defined in combination with scores for other desiderata) such that surpassing it will prohibit a function from counting as a law. It's unclear, however, that the revisionist has similar defences for the desiderata of tractability, employability for partial modelling, error-tolerance, breadth and modularity. Our current best guesses for the actual laws of physics are impressively simple in number and syntactical complexity. They do, however, admit non-computable solutions 
(e.g. the Schrödinger wave-equation, Pour-El \& Richards, 1981, and the NavierStokes equations). Also, a whole body of work on chaos is built around the idea that some systems are very much error-intolerant. Most physicists today also believe that the fundamental laws of physics concern systems of which there is technically only one (the entire universe) which counts as truly isolated. If breadth, modularity and partial modelling were to concern only purely isolated systems then the laws of this world would score a minimum for all three desiderata. It's hard with these observations to hand to defend the idea that any of these desiderata establish a threshold (determined in combination with scores on other desiderata) beyond which generalisations could not count as laws.

Perhaps the revisionist can still coherently maintain that the revisionary desiderata nevertheless play a role in the competition for best systems. The winning systems, they might say, are able to score less than a maximum, even a minimum, for some desiderata so long as there are high scores for others. Alternatively, the revisionist might propose that revisionary desiderata only come into play when sufficient strength and simplicity is reached. ${ }^{19}$ They might also define a notion of 'quasi-isolation', as Hicks does, to circumvent the fact that no sub-system is precisely isolated. But one should wonder whether these are really plausible things to say.

It is common among physicists to think that the usability of a generalisation is irrelevant to its lawhood: 'fundamental physics doesn't care that much about us' so say Susskind \& Friedman $(2015,104)$. And if the laws did 'care about us', in the sense of tending to exhibit error-tolerance, partial modelling, tractability, breadth and modularity as constitutive characteristics, then it would be curious why these features don't get mentioned alongside simplicity and comprehensiveness when physicists like Feynman and Einstein hold forth on what it is they think physics is all about. 'So much for the off-hand remarks of physicists,' one might be tempted to say. But the revisionists' endorsement of the pragmatic turn makes it particularly difficult for them not to take notice of physicists remarks, assuming they are representative. The very idea of the pragmatic turn is to develop an account of laws according to which the virtues of theory-building in physics are built in as constitutive features.

Revisionists should therefore ask themselves whether it really is plausible that, if there is a choice between one system that is best-achieving according to the canonical desiderata (making 'the irreducible basic elements as simple and as few as possible without having to surrender the adequate representation of a single datum of experience') and some alternative system which scores higher on some further revisionary desideratum, then it could be the latter whose theorems really provide the laws. There is at least some indication from philosophical thinking about counterlegal possibilities, the comments of leading physicists about their own field, and from the laws physics has in fact discovered, that the answer is 'no'. In that case, it is more than a mere possibility that the proffered revisionary desiderata play no role in settling what it is to be a law at all.

By way of contrast, compare this possibility with something which I take clearly not to be possible: that strength and simplicity not only don't play a

\footnotetext{
${ }^{19}$ Woodward (2014), already suggests something might be going on for simplicity, though see my response on p.5.
} 
role in settling the best systems but could permissibly score zero for some bestachieving systems. The Humean has principled reasons to deny that all the theorems of a system which scores nothing for either simplicity or strength can be the laws. Lewis hinted at what such systems would have to be like. 'Simplicity without strength can be had from pure logic, strength without simplicity from (the deductive closure of) an almanac' (1973, 73). But generalisations deduced from almanacs provide no distinction between accidental and lawlike regularities, and generalisations deduced from logic alone would all be metaphysically necessary. Since the very purpose of the Humean approach to laws is to find a way to distinguish lawlike from accidental regularities in a manner which retains the former's contingency, systems which entirely lack simplicity or strength are categorically not systems all of whose generalisations are laws.

So, revisionists uniquely face the problem of explaining how their desiderata can be involved in a trade-off (or criterial point-scoring) for best systems when it seems they need not be represented at all. At the very least, the revisionary desiderata just don't seem so crucial to lawhood as the canonical desiderata. Perhaps that's something they will ultimately accept, but this is a far cry from the bold revisionary programme they have been taken to be promoting.

\subsection{Summing up the revisionary proposals}

In this section I've enquired as to whether it's really plausible that any of the revisionary desiderata are involved in assessing the best-achieving systems, which (for the Humean) give us the laws. Looking at the what we want from laws and the sort of thing we could have access to shows that many of the revisionary desiderata proposed for facilitating these features are ill-motivated. Some other proffered desiderata are perhaps not so easily undermined, particularly Hicks's and those offered on account of their ability to facilitate ease of use. Nevertheless, revisionists should face up to the fact that there is a strong case to be made that physics is just not about pursing generalisations with these revisionary characteristics. Strength and simplicity remain the most justifiable and continually emphasised desiderata physicists look for in their laws.

\section{Conclusion}

The pragmatic turn in BSAs is motivated by the need to avoid to 'ratbag idealism'. It acknowledges that the desiderata which determine the standards for which systems come out best, and hence what the laws are, is a pragmatic affair. This should have come as no great surprise to Humeans, since the widely-told parable of God's concise encyclopaedia given to us for our own comprehension makes it evident that BSA takes laws to be exactly something to help us. The pragmatic turn is, therefore, well taken. However, the plethora of counterexamples to canonical BSA suggest that the desiderata of simplicity and strength must be revised, and this has instigated a 
flurry of proposals justifying the inclusion of new desiderata on pragmatic grounds. In the foregoing, I've aimed to show that this revisionist project is likely misguided. First, there are reasonable responses to the counterexamples from within the canonical framework, so revisionism is probably unnecessary. Second, the pragmatic virtues attributable to laws do not clearly motivate any of the proffered revisionary desiderata. There are likely no non-canonical pragmatic virtues which necessarily factor into the competition for best systems which determines the laws, and moreover, the canonical desiderata seem alone able to explain the pragmatic virtues laws in fact exhibit.

Acknowledgements I would like to thank Mike Townsen Hicks, Chris Dorst, Francesca Bellazzi, Samuel Kimpton-Nye, John Roberts, Vanessa Seifert, Tuomas Tahko and two anonymous reviewers for their extremely helpful comments to this paper's drafts.

Funding The research for this article has received funding from the European Research Council (ERC) under the European Union's (EU) Horizon 2020 research and innovation programme, grant agreement No 771509 ('MetaScience').

\section{Declarations}

Conflicts of interest The author is Assistant Editor for the European Journal for Philosophy of Science. This article was nevertheless submitted blinded and referees advised editors that it be accepted without knowledge of the author.

Open Access This article is licensed under a Creative Commons Attribution 4.0 International License, which permits use, sharing, adaptation, distribution and reproduction in any medium or format, as long as you give appropriate credit to the original author(s) and the source, provide a link to the Creative Commons licence, and indicate if changes were made. The images or other third party material in this article are included in the article's Creative Commons licence, unless indicated otherwise in a credit line to the material. If material is not included in the article's Creative Commons licence and your intended use is not permitted by statutory regulation or exceeds the permitted use, you will need to obtain permission directly from the copyright holder. To view a copy of this licence, visit http://creativecommons.org/licen ses/by/4.0/.

\section{References}

Albert, D. (2015). After physics. Harvard University Press.

Ayer, A.J. (1999 [1956]). What is a law of nature? In: M. Tooley (Ed.), Laws of nature, causation, and supervenience (pp. 1-52). Garland Publishing

Beebee, H. (2000). The non-governing conception of laws of nature. Philosophy and Phenomenological Research, 61, 571-594.

Braddon-Mitchell, D. (2001). Lossy laws. Noûs, 35(2), 260-277.

Cohen, J., \& Callender, C. (2009). A better best systems account of lawhood. Philosophical Studies, 145, $1-34$.

Davidson, D. (1995). Laws and cause. Dialectica, 49(2-4), 263-279.

Demarest, H., \& Hicks, M. T. (2020). Isolation, not locality. Philosophy and Phenomenological Research, $00,1-13$.

Dorst, C. (2019). Towards a best predictive system account of laws of nature. British Journal of Philosophy of Science, 70, 877-900.

Earman, J. (1986). A primer on determinism. D. Reidel Publishing Company. 
Eddon, M., \& Meacham, C. (2015). No work for a theory of universals. In B. Loewer \& J. Schaffer (Eds.), A companion to david lewis (Chapter 9, pp. 116-137). Wiley-Blackwell.

Einstein, A. (1918 [2002]). Principles of research: Address for Max Planck. In: The Collected papers of Albert Einstein (vol. 7). Princeton University Press.

Einstein, A. (1934). On the method of theoretical physics. Philosophy of Science, 1, 163-9.

Feynman, R. (1967). The character of physical law. Cambridge University Press.

Friend, T. (2016). Laws are conditionals. European Journal for Philosophy of Science, 6, 123-144.

Friend, T. (forthcoming). How to be humean about symmetries. The British Journal for the Philosophy of Science. https://doi.org/10.1086/716924

Goodman, N. (1954[1983]). Fact, fiction and forecast. Harvard University Press.

Hall, N. (2015). Humean reductionism about laws of nature. In B. Loewer \& J. Schaffer (Eds.), A companion to David lewis (Chapter 17, pp. 261-277). Wiley.

Hartle, J. (2005). The physics of now. American Journal of Physics, 73, 101-109.

Hicks, M. T. (2018). Dynamic humeanism. British Journal of Philosophy of Science, 69, 983-1007.

Jaag, S., \& Loew, C. (2020). Making best systems best for us. Synthese, 197, 2525-2550.

Lange, M. (2007). Laws and meta-laws of nature: Conservation laws and symmetries. Studies in History and Philosophy of Modern Physics, 38, 457-481.

Lange, M. (2009). Laws and lawmakers: Science, metaphysics, and the laws of nature. Oxford University Press.

Lange, M. (2011). Meta-laws of nature and the Best System Account. Analysis, 71(2), 216-222.

Lewis, D. (1973). Counterfactuals. Blackwell Publishing.

Lewis, D. (1983). New work for a theory of universals. Australian Journal of Philosophy, 61(4), 343-377.

Lewis, D. (1986). Counterfactual dependence and time's arrow. In: Philosophical Papers II (Chapter 17). Oxford University Press.

Lewis, D. (1986 [1980]). A subjectivist's guide to objective chance. In: Philosophical Papers II (Chapter 19, pp. 83-132). Oxford University Press.

Lewis, D. (1994). Humean supervenience debugged. Mind, 103, 473-490.

Loewer, B. (1996). Humean supervenience. Philosophical Topics, 24, 101-127.

Loewer, B. (2007). Counterfactuals and the second law. In H. Price \& R. Corry (Eds.), Causation, physics, and the constitution of reality: Russell's Republic Revisited (pp. 293-326). Oxford University Press.

Loewer, B. (2007). Laws and natural properties. Philosophical Topics, 35(1/2), 313-328.

Loewer, B. (2012). The emergence of time's arrows and special science laws from physics. Interface Focus, 2(1), 13-19.

Maudlin, T. (2007). The metaphysics within physics. Oxford University Press.

Pour-El, M. B., \& Richards, I. (1981). The wave equation with computable initial data such that its unique solution is not computable. Advances in Mathematics, 39, 215-239.

Ramsey, F. (1978 [1928]). Universals of law and fact. In: D.H. Mellor (Ed.), Foundations: Essays in philosophy, logic, mathematics and economics. Routledge and Kegan Paul.

Roberts, J. (2008). The law-governed universe. Oxford University Press.

Schrenk, M. (2014). Better best systems and the issue of CP-laws. Erkenntnis, 79, 1787-1799.

Susskind, L., \& Friedman, A. (2015). Quantum mechanics: The theoretical minimum. Penguin.

Woodward, J. (2014). Simplicity in the best systems account of laws of nature. British Journal of Philosophy of Science, 65, 91-123.

Publisher's Note Springer Nature remains neutral with regard to jurisdictional claims in published maps and institutional affiliations. 